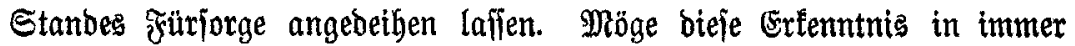
weitere Sreife oringen und reidje Früchte tragen.

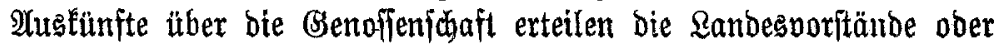

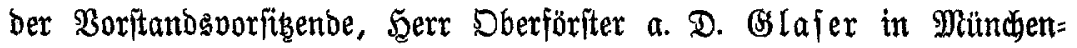
Pajing.

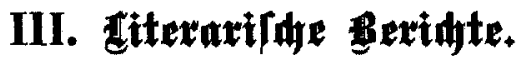

Mr. 13.

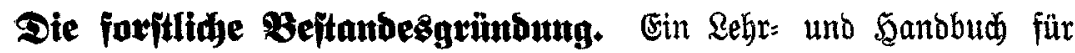

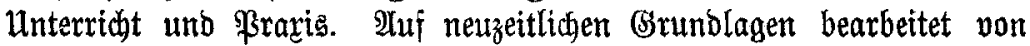

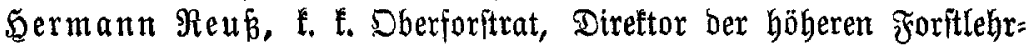

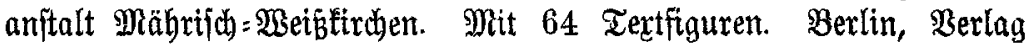
von Şulius Springer, 1907. 398 S. Breis $8 \mathscr{H}$, geb. 9,20 $\mathscr{M}$.

Mit bem vorliegenden gräperen Wert mendet fid ein Mann an bas

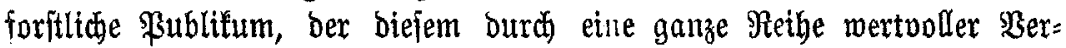
offentlichungen bereits in vorteil'hafter $\mathfrak{B e i j e}$ befannt ift und jobn um

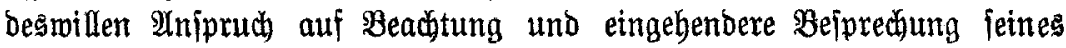
\$ुubes erheben barf; eine jolde möge ifm benn nadjfteheno gemiomet jein.

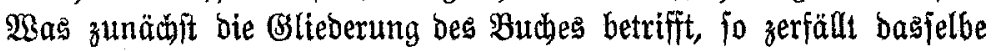

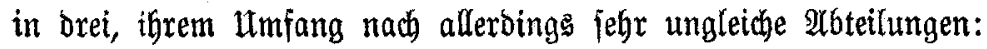

I. Abteilung: Die Beitandesgründung burch Samen (348 Seiten)

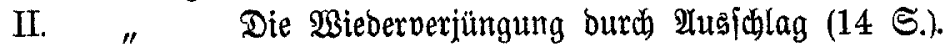

III. " Die angewanote Sebre $(27$ S.).

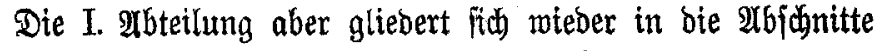

1. Die natürlitige ßSeriüngung (38 $\mathfrak{S}$.),

2. Die Künitlitje Beitandesgründung (310 S.)

und in ber Befprechung ber leketeren liegt fonadh ber Scherpuntt bes Bubles. Diejer umfangreide alofdhitt behandelt bas Saatgut, bie $\mathfrak{B e}=$ ftandesgrünoung burd) Saat uno burch Pflanzung, und fügt in einem weiteren Rapitel "Die Urbarmadbung von ber ummittelbaren Mufforitung

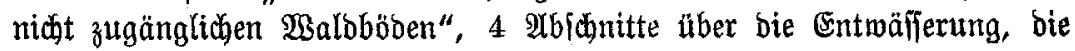
Flugjanbbindung, bie melibrierende Behandlung von Waloböben mit Robhumusauflagerung, Den Drtitein uno jeine Urbarmadumg an.

Benden wir uns mun ben eirzelnen 9 bjobritten $z \mathfrak{u}$, jo tritt uns in

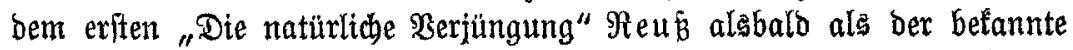

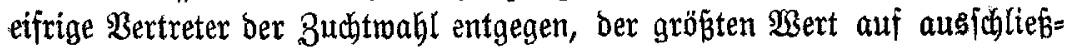
liche Bermendung Geiten, won tabellojen Mutterbäumen ftammenden Samens legt. Reup wertritt biejen Stanopunft eingehento in bem $\S 15$ 


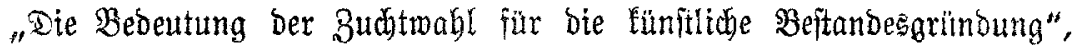
aber ex ziegt fich audh als roter Faben burch bas ganze $\mathfrak{B u t h}$, uno fo forbert $\Re$ en $\beta$ benn jobon bei ber natủrlichen Berjüngung foharfe Mufterung aller Samenfäume, Bejeitigung nidgt nur bireft tranfer unb fihabhafter şöume, fondern aud ber Giabelmüble, ber Stämme mit tief angeiebten

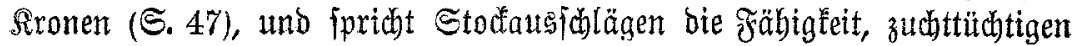
Samen zut erzeugen, ab. Mix möbten glauben, bak er hierbet bod zu weit geht!

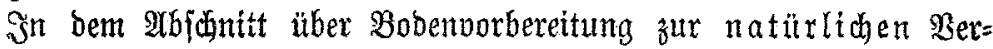
jüngung $(\$ 6)$ baben wir die Erröăhnung der neueroings viel benukten Rofleggen permißít, und als neues - aber boch mobl febr jelten an= gewendetes - Mittel bas 2Hbbrennen hinberlicter Bodenüberzüge ("Unter= Iandbrennen") unter einem zu veriüngenden Seitand angegeben gefunben. Sso in einem Beitand fold' ftarfe ütberzüge vorbanden, wirb man wobl zumeift auf natürliche $\mathfrak{B}$ eriüngung verzichten müffen!

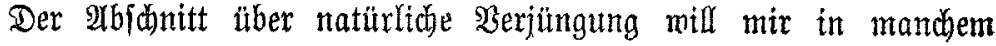
überhaupt etwas fnapp behandelt eridheinen: fo in bent $\$ 8$ "Die Schirm= veriüngung im Saumidlag", bie neuerbing Frofeffor $\mathfrak{B} \mathfrak{a} \mathfrak{g} \mathfrak{n} \in \mathfrak{r}$ in Tibingen fo eingehend bebandelt bat, 1 ) in Dem $\$ 14$,Die Machbefferungg:

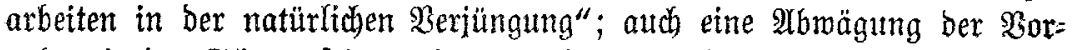
teile wie ber Sdhattenleiten, pine Ingabe der Fälle, in welchen bie natür= liche voer bie füntliche Berjüngutg vom wirtfdaftlidyen ober finanzillen Stanopunft aus vorzuziehen jei, würbe am Plate geweien jein. Es ents fpridbt bieje kürzere $\mathfrak{B}$ ebanblung ber Stellung, welche $\Re$ eú und 91 bezäglich ber fümftlichen 9 Serjüngung und ipeziell ber Beftandes: gründung burch Fflanzung einnimmt: er meift leb̧terer unbebingt bie erfte $\Re$ olle im Berjüngungsbetriebe zu, an melcher burch eine überwälti= gende ₹rülle von Erfahrungen belegten Rehrmeinung leine Qlutorität zut

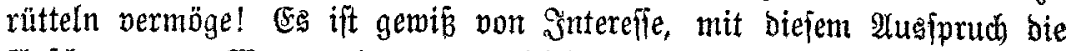
Mnjdaumnen $\mathfrak{B a g n e r}$ 'su vergleidhen, ber in jeinem oben zitterten

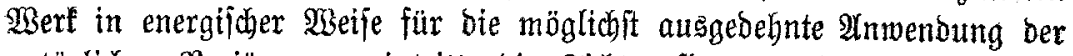
natürliçen Berjüngung eintritt, bie Fithtenpllanzung in ihrer üblitben Art als eine "Beleibigung ber Ratur" verwirft! Es bürfte wohl autd Gier bas Midhtige in ver Mitte liegen!

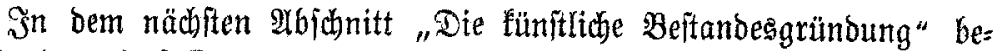
ipricht ber Berfafjer zunädjit eingebeno das Saatgut unb beffen $B e=$ fibaffung und legt fetne weitgehenden Anfiprberungen bez. Der 3 udbtwahl

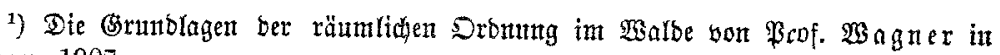
Sitbingen 1907 .

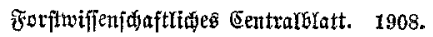




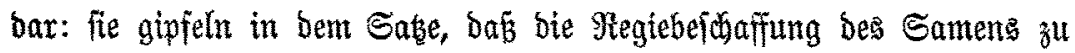
ben erniteften und vornehmiten Fffichten bes forithaushaltes gehöre wir fügen bei: aber aud za ben wohl in vielen Făflen fäjwer zat er= füllenten!

Sobant wiro bie Beftandeşjaat in allgemeinen gewürbigt unb

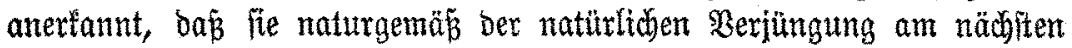

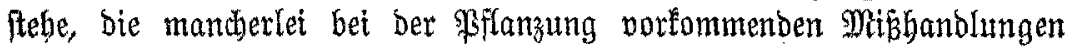

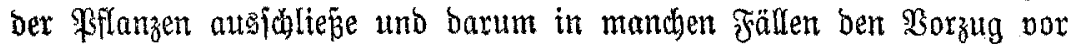
Der sfflanzung verbiene - gletdiwohl jet fte der exfolgfichereren Pflanzlung nachzuftellen. Shre Bepprectung ifit beshalb auch in engeren Grenzent

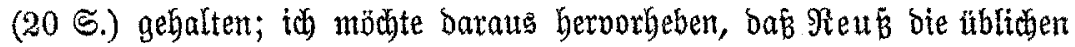
Reimproben vermirft und bie Iopfprobe forbert, und baß auth bie $\mathfrak{A} n=$ wenoung ber Memnige verworfen wirb. Bei ben gelegentlici ber Boben=

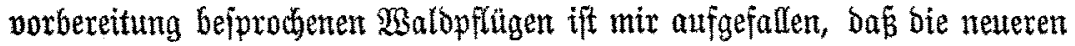

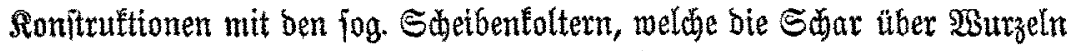
uno Steine binwegheben, nidbt erwähnt find.

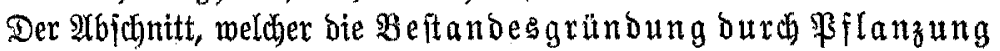

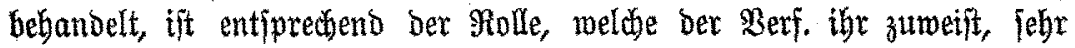

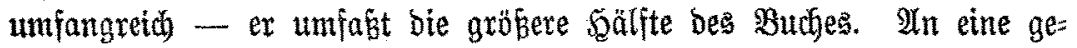

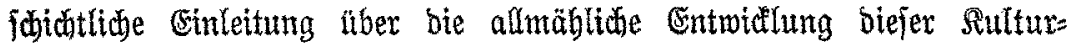

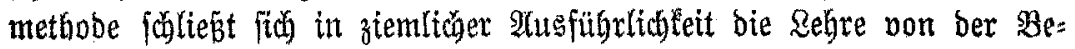

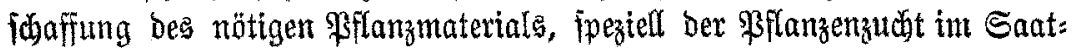
uno \$ffanzamp; biejelbe entipridft bem Stand ber Gegenwart, boch mỏdten wir uns geftatten, etnigen Bebenten 2 usbrud zu geben.

Bei ber Bodenvorbereitung für Saatfämpe miro angegeben (ङ. 116),

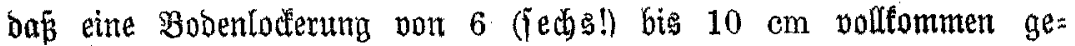
nüge! Dagegen ift ebenio entifhteden (Etriprutu za erbeben, wie gegen bie S. 138 angegebene Tiefe ber Eaatrillen, weldhe für Grob̆torn= jamen $2-3 \mathrm{~cm}$, für Rleintornjamen ebenjo viele Millimeter betragen foll; Cidfeln, Edelfaftanten beclit man jedenfalls tiejer als $2-3 \mathrm{~cm}$, uno

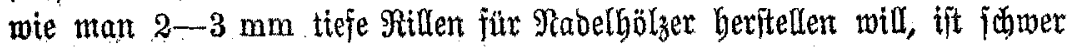
exftnolid: $10 \mathrm{~mm}$ tief bedt man beren Samen bod wohl uberall.

Uls Săeapparate werben auffallendermeife (S. 139) bie ganz vet:

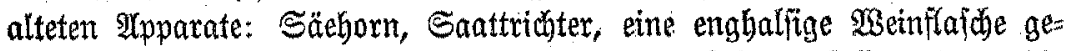

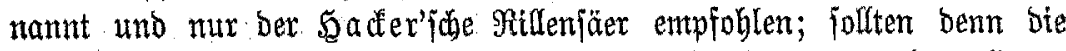
portrefflitoen Säeapparate pon Eblinger unb Görmann bem Serrn

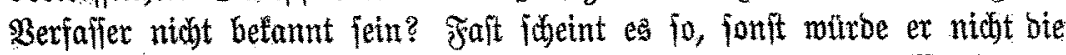
Sanbjant empfeblen, bie nadi unjerer Injedt bireft als rüdftänbig zu bezeidfinen ift!

(Einfprud muß aber vor allem erboben werben gegen bie von $\Re \mathfrak{e} \mathfrak{B}$ 
empfohlenen Samenquantitäten. Madjom er fith (S. 136) fehr entfobieden

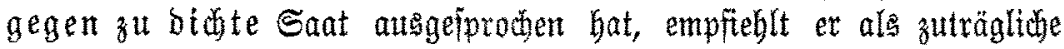

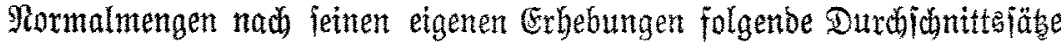
für 1 qm: Eithe $1,80-2,00 \mathrm{~kg}$, Jide $7 \%-80 \mathrm{~g}$, Riefer $70-80 \mathrm{~g}$,

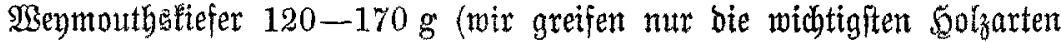
beraus). Dae würbe jonady für $100 \mathrm{qm}=1$ a geben: für (siche 180 bis $200 \mathrm{~kg}$, fïr Fithte $7,50-8 \mathrm{~kg}$, Riefer $7-8 \mathrm{~kg}$, $B$ Beymoutthgltefer $12-17 \mathrm{~kg}$ - bas fins burbjidnittliti bie vierfachen Mengen, wie fie in ber Siteratur ${ }^{1}$ ) als üblich angegeben werden; wie fommt ein fo er=

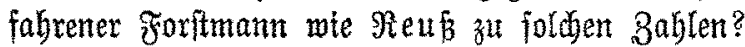

Den itber bie Berpflanzung ing Freie Gandelnden umfangreiden

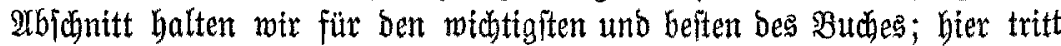

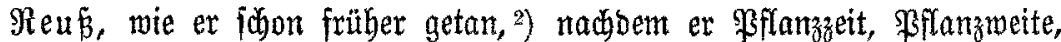

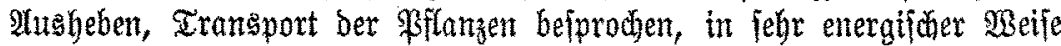

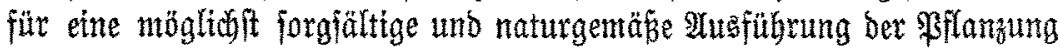
ein. Er weift barauf bin, wie vielfach burch forglojes Pftanzen, burnt Einpreffen in enge Pflanżlödher, Durch zu tiefes Eimpflanzen gejündigt uno bie ganze 3 utunft berart begrünoeter seftänbe gefährbet miro, zeigt

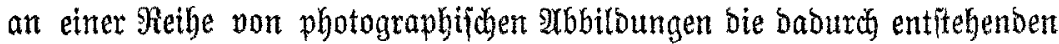
Wurzelmifbiloungen unb warnt in cinbringlicher $\mathfrak{B e t j e}$ vor folden zehlern. Man wiro ihm bier unbebingt beiftimmen uno jeiner Warmung möglidift Berbreitung wünjoben miiffen.

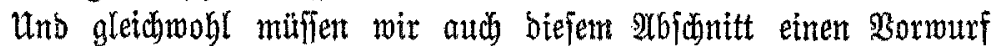

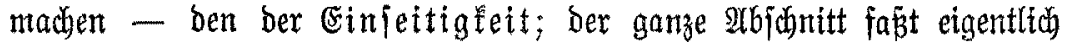

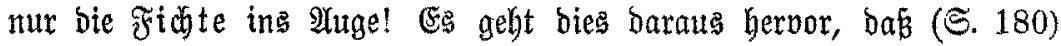
ausgeiprodjen ift, ber grope אulturbetrieb greife nidft unter bas zwei= jährige ßfanzenalter herab; baj (S. 225) bie "Berätemethoden" uno

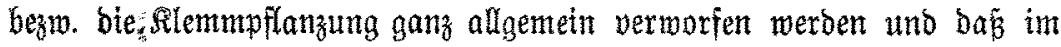
weiteren eben $\mathfrak{n u r}$ bie Fffanzung mit ber Sand in weite, mittelft ber Szacte gefertigte Fflanzlöber beforieben wirb. Demgegeniuber jet auf bie Millionen einjähriger Siejern bingewiejen, bie alljährlid mit beftem Erfolg in Deutichland und wobl aut Diterreich mittelft Rlemmplanzung verpflanzt werben - baran barf bod ein von Beftandsgründung han: belndes Spezialmert nidt vorübergehen! Sid) balte bie నilemmpflanzung 1 jähriger Riefern, Sdhwarghtefern, Eichen, Ebelfaitanien in gut ge= loçerten Bo oen, mit weiten Pflanzlobdern forgfältig ausgeführt für

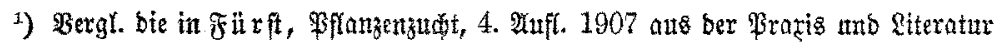
mitgeteilten Bablenangaben.

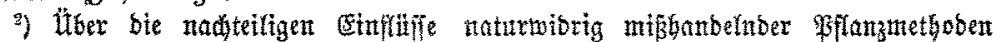

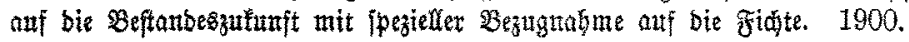




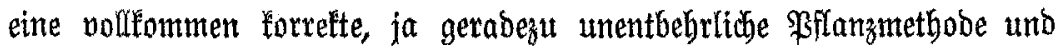
aud $\Re$ Rú gibt fpäter wieberbolt beren 2 mmenbbarfeit, jogar für bie

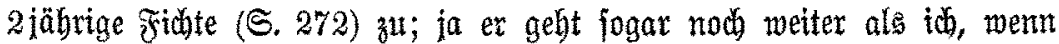
ex 5.366 bie Anwenbung ber \&lemmpflanzung fogar nod fitr $3 j$ ährige (Et)ienfämlinge geltatten will, wogegen ich entjobieden proteitteren möbte!

SBsenn aber bie Slemmpflanzung als zuläfftg enflärt wirb, bann

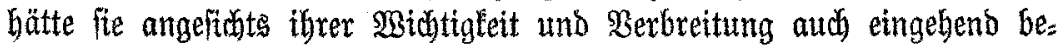
fprochen, ibre ridhtige $\mathfrak{A}$ ugfubrung bejörieben merben jollen; ich balte bies für einen entidiedenen Mangel bes fonit gewín guten $\mathfrak{B e r f e s . ~ - ~}$

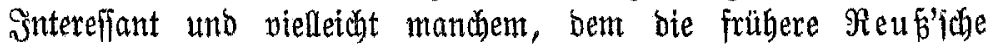

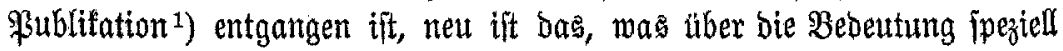
ber Bejerpfrieme als Stidftoffiammier gejagt witb.

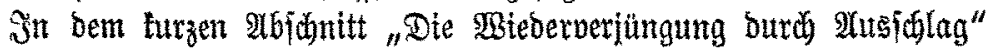
wirb auffallenderweife nur ber Miederwald beiprochen, ber Mittelwald gar nicht ermähnt, obwohl gerabe biejer bei jebesmaligem atbtrieb ganz

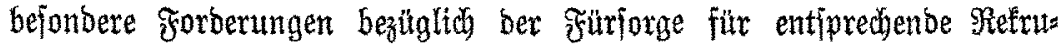
tierung bes Dbergolzes burd Fflanzung paffender solzarten ftellt.

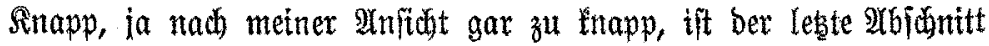
"Die angemandte Rebre" gefajt, in weldem mun bie einzelnen Szolzarten nad je 3 Gefictispunten hin: natürlthe Beriüngung, Beitandesjant uno Beitanbespflanzung - befprodien werben; bei ben Raubbölzern gejellt

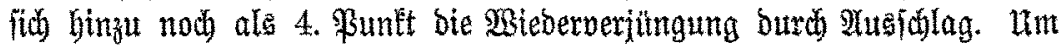

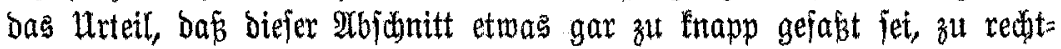

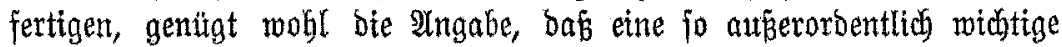

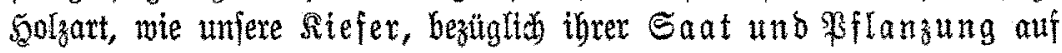
36 Beilen abgefertigt wirb! Soffte bies in einem $\mathfrak{G a n b b u d ~ f u t r ~ b i e ~}$ Braris ausreichend pein? -

Ş) Gabe, wie aus \$orftebendent Gervorgeben bürfte, bas umfang= reiche Buth gründiti und mit fritifbem Blid burchftubiert uns - sine ira et studio - alles bervorgeboben, was meinen eigenten IInfdauungen

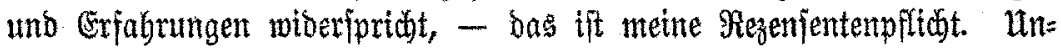
möglid fann ich aber aud alles betonen, wortn id mit bent Alutor übereinitimune, und defien ift gemí weit mebr; ben Rejern bes Bubles

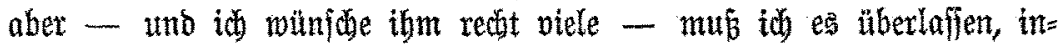
wietweit fie meinen bejotbenen Einwenoungen zuftummen.

\section{Dr. F゙̈} II. 1903.

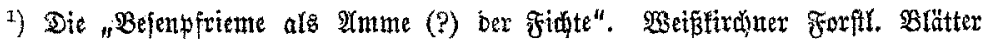


शr. 14.

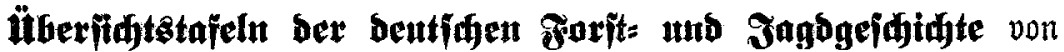

Dr. Rarl $\mathfrak{B i m m e n a t e r ~ u n o ~ D r . ~ S e i n r i d ~} \mathfrak{B e b e r . ~ B e r l i n , ~ b e i ~}$ Springer, 190\%. Freis 1 All.

Die vorliegenden 4 Iafeln find in je 9 Spalten gegliebert mit ben überibriften: Dueflen; Beographiche Berbreitung ber Szauptholzarten;

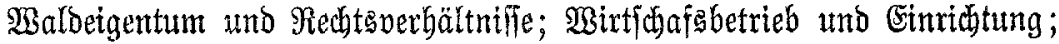

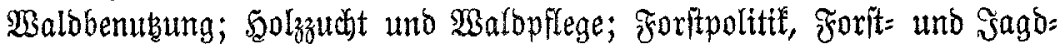
itrafmeien, Forftichus und Berwaltunt; Forftliche Interrictets= unto Bil= Dungsanftalten; Frorftltbe und jagdliche Riteratur. In 57 \$aragraphen werben bie widbtigften Tatjachen be: Forit: unb Sagogejicichte aufgeführt. Die Şerren Berfaffer baben bie Tafeln zujammengeftellt, um ihren \$örern bas Siadjiobreiben zu erleidhtern und un zum Stubium ber Foritgejobihte

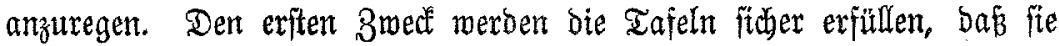
aud in ber zmeiten Sinficht expolgreich leien, ift felhr zu wünjohen. Sidjer werben fite ben Eramenăfandioaten als Repetttorium jehr willtommen jein.

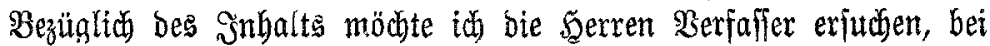

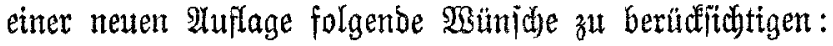

క̌n $\S 9$ unter ben Duellen auch ber Drts= und Gewanntamen zu geoenter, da bieje von immer größerer Bebeutung merben, je mehr gutes Rartenmaterial vorliegt und burdh bittorifche Drtsperzeitaniffe bie alten Namensformen betannt werden.

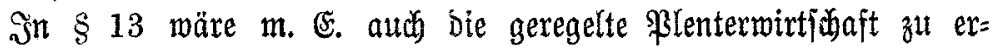
wähnen, bie wenigitens in Sübweftbeutidhland als Bammald föbn um 1450 eine grofie $\mathfrak{B}$ erbreitung bejaj.

§m $\S 16$ jollte als 3eitpunft ber erften (Fia)elfaaten ftatt 1491 zu Seligenftabt, 1398 zu Franffurt a. Th. gejekst werben (vergl. Fellner, Beitrag zur (Jejdichte des Stadtmaldes von Franfiurt a. M. 1895). शluch wäre hier vielleidjt ber Eidhenpflanzung zu gebenten, für bie im Freiburger Stabtwald bereits 1457 ein Eichenfamp beftand. (Gserber,

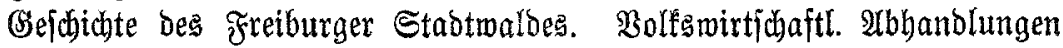

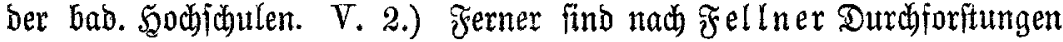
bereits um bie Mitte bes 15. Jjabrbunderts im Franffurter $\mathfrak{B a l b}$ aus: geführt worden. Enolid dürfte die Babl 1494 für bas Engelmannabuch

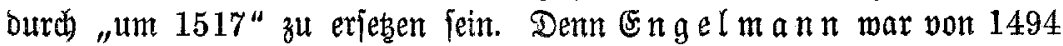

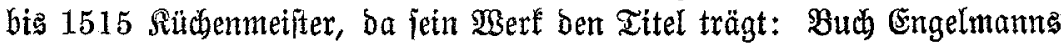
etwann gewejenen ... Rüchenmeifters...., ijt es aljo erft nad biejer Beit verfábt. Damit ftimmt aud eine Stelle im Iert bes Buches, bie beribtat, baj ein Wiejentnedft lutherifid geworben jei, was fich boch fritheftens 1517 ereignet baben $\mathfrak{f a n n}$, 


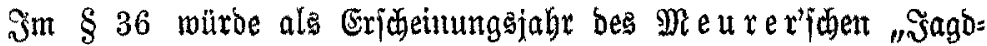
unb Forftredsts" wohl beffer $15 \% 4$ genanni. Denn bie 1561 erforienene

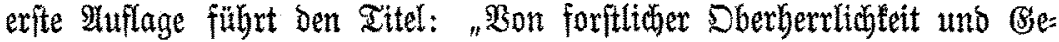
rechtigfeit", fie bebanbelt aud faft lebiglio bas Sagbregal. Erit in ber zweiten 2 luffage bat bas sere ben neten Iitel, bie Geftalt und ben

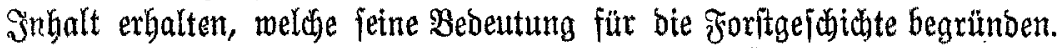

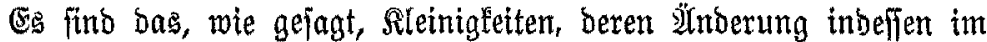
Sntereffe ber $\mathfrak{T} a f e l n$ felbft liegt. $3 \mathfrak{u m}$ Saluffe fei mir geitattet, audi

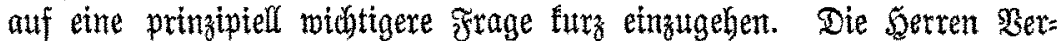
faffer haben bic üblide Seriobenteilung: bit 911, 911-1500, 1500 bis um 1750, nach 1750 beibebalten. Fir bie Foritgeidichte bildet aber bas

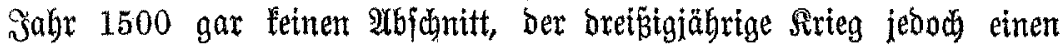

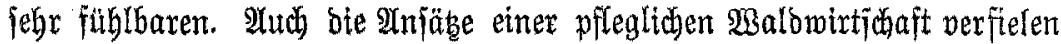
unter jeinen Stürmen, um in ben meiften Gegenden erit viel jpäter wieber zu exfteben, bie finanzieflen Söte, bie ber Rrieg Gervorrief, waren

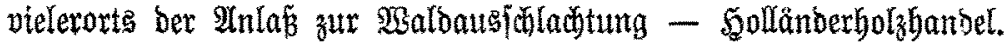

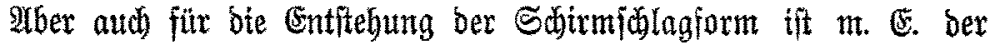
lange Sirieg won erbeblider Bebeutung gewefen, ba fich währent desfelbent

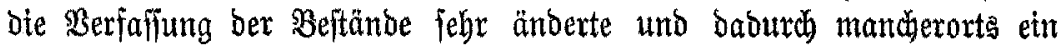
Berjagen ber alten Mittelwalbidhlagwirtidaft verurjadht worben ift. Eine

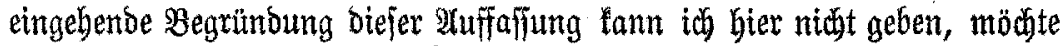
aber boc eine entipredende Ânoerung angeregt baber.

Sarlstube, im Nopember 1907.

Dr. $\mathfrak{2} . \mathfrak{g} \mathfrak{a} \mathfrak{a} \mathfrak{r} \mathfrak{a} \mathfrak{t}$.

Nx. 15.

\section{ïber bie Fortp/angungsuexhältnifie ber rinbentrütenben}

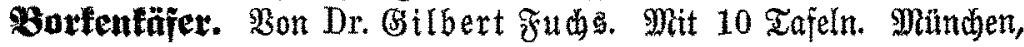
Serlag von Reinharot, 1907. Pretg 6 Af.

2luf Grano langiährigex Beobadtungen in ber freten Ratur uno zahlreicher 3uchtnerfudbe jobildert Berfaffer unter fritifider Bärbigung Der älteren unt netteren Riteratur bie Frortpffarzung ber Borfenfäfer, in

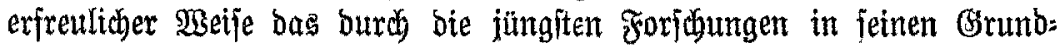
linten entworfene $\mathfrak{B i l d}$ meiter augfülfrend uno burds mande neue $\mathfrak{T} a t=$ fache ergänzenb. Um eingebenoften bebantoelt er 1 . ben "Machfrä" ber

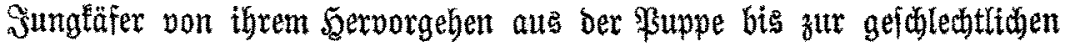
Bolleife und Etablage; 2. Den auf bie Brutablage folgenden bis zur erneuten Funttionsfäbigfeit ber erjö̈ppften Fortpflanzungsorgane fort= gejekten jogenamnten "ßRegenerationģra nad) ber einfachen und ooppelten Generation ber einzelnen $\mathfrak{A}$ rten bezw. 
(Sruppen. Für Hylesinus fraxini und Ips typographus fann in $\mathfrak{B}$ ezıg auf bieje Puntte bas vom Berfaffer entworfene Rebensbillo in jeinen Sauptzïgen als abgefdlofjen gelten; aber aud für zablreiche andere Urten bringt Berfaffer neutes Material. Dhne auf bie zaulle bex (Sinżel=

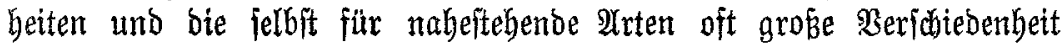
ber Rebensmeife einzugehen, möbte Referent nur bas Folgende heroor= Geben: $2 l b g e j e h e n$ von ben Eccoptogafter = Urten und einigen Feglefininen,

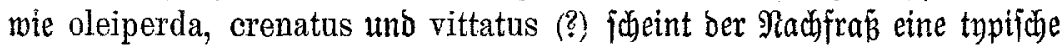
Erjheinung zu jein, wenn auch je nach ber betreffenden Spezies veridjieben

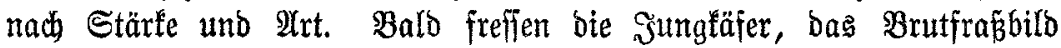
verändernd, ia oft unfenntlich madjend, an ifhrer Geburtsftätte, bald nach Berlafien berjelben an neuem Matertal: $\mathfrak{B u r z}$ eln, Trieben, frijher Minde, und zwar teils nach voraufgegangenem $\mathfrak{F} r a \dot{B}$ am Geburtsort, wie viele Spinen, teils ohne jolchen, wie bie Maldgärtner und Hylesinus fraxini.

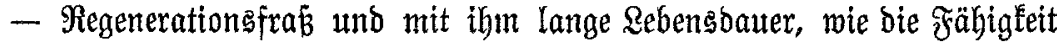
zur Ablage einer zweiten, vielleidjt felbịt mehrfacher Brut wiro in $B e=$ ftätigung früberer $\mathfrak{A}$ ngaben ober nach eigener Beobachtung bezw. Bucht

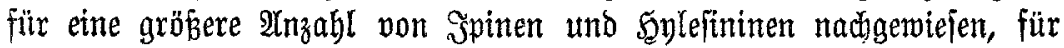

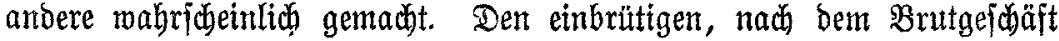
alsbald fterbenden Eccoptogafterarten fehlt er. IUtd bie Unteriudung

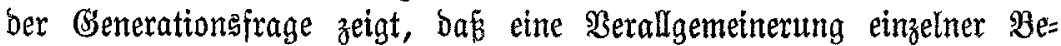
obachtungen unzuläfïg ijt. Stets einfache Generation befthen z. F. Die Walogärtner, Hylesinus fraxini, Dendroctonus micans, bie $\mathfrak{S B u r z}_{3} \mathrm{el}=$

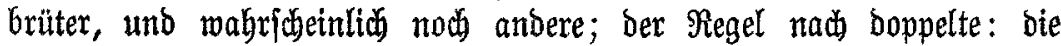
Eccoptogafterarten, vielleidit mit $\mathfrak{A}$ usnahme von Ratzeburgii; ie nach

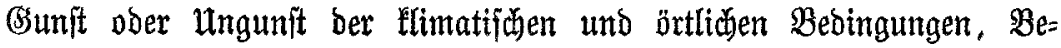
fonmung, Schatten, Trodenheit, Feuchtigteit, Doppelte ober einfacte: bie

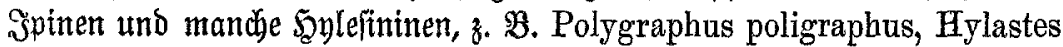
glabratus unb palliatus, ujw.

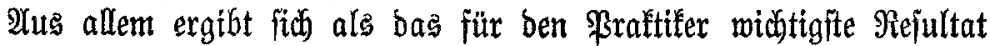
bie auf anderer (Srundlage erneut feitgeftellte Tatiadie ber fteten Schmärm= und Brutmöglichfeit ber \$orfenfäfer. Berfaffer betont autsorïaflid, ba

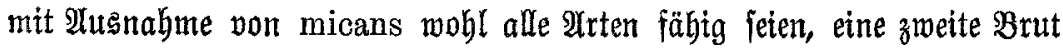
abzufeken, fei es, baj fie boppelte Generation baben, dober bei einfacter Generation mebrbrütig find, ober endith aus beiben (siünden.

Die bem $\mathfrak{B e r k d j e n ~ a u f ~ z e g n ~} \mathfrak{I} a f e l n$ beigegebenen, nach \$hotographien

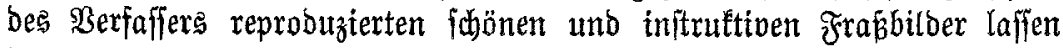
ben Prets von 6 ath nidgt zu hoch ericheinen.

Dr. S. 
Nr. 16.

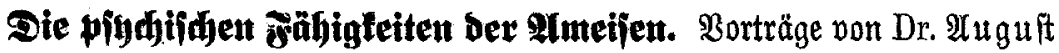

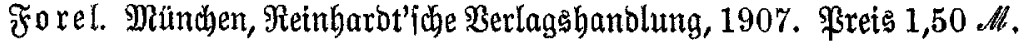

Das Froblem ber tierifden Sindje ift viel umitritten. Albgejeben von bem Standpuntt bes naiven Beobadhters, ber in bent wunderbaren "Staatenleben" ber 2Imeijen und Bienen ein 2 Mnalogon bes menifdlichen Staates zu erblicten meint und $\mathfrak{B}$ asmann's naturmiffenjibaftlid nicht

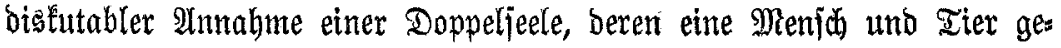

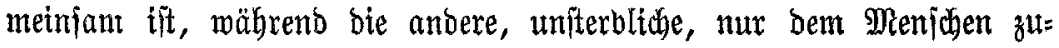

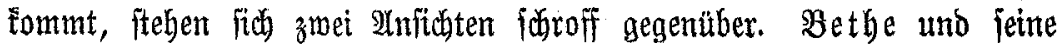
$\mathfrak{U n h a ̈ ̈ n g e r ~ L e u g n e n ~ j e b e ~ M o ̈ g l i c h f e i t , ~ b e i ~ n i e b e r e n ~} \mathfrak{I}$ ieren pind hijhe Dualitäten zu ertennen, uno ertlären alle SBirbellojen jür jeelenlofe Reflexmajodinen.

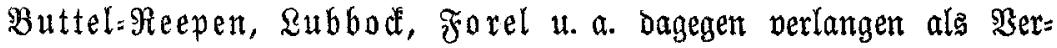

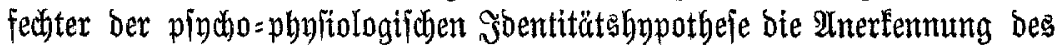
induttiven 2 (nalogiefdluffes als einer naturwiffenjojaftlid beredigtigten Methobe. Wenden wir bieje unbedentlith bei unjeres (Sleidjen an, ob= wohl boch ein S̈eber fich nur jeines éigenen Bemubtjeins ohne weiteres

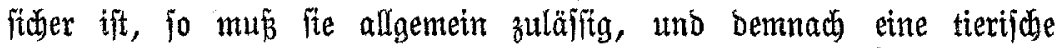

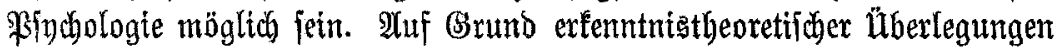
unb eines reichen, burch Beobachtungen und Erperiment gemonmenen

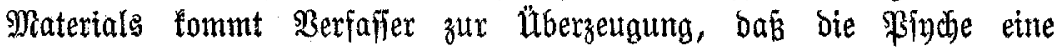
urfprïnglide (Etgenjidaft alles tierifden Rebens ift, zwar unenolid ver= jajieben in ifrer Entfaltung, aber ber bes Menjoben wejensgleidy. WBie bie lörperlitben, laffen fich aud bie Seeleneigenifdaften bes Menichen aus benen ber höheren Iiere, und bieje wieber aus benen nieberer Lebewejen ableiten. Die Bewetje für feine $2 \mathfrak{2 n j}$ jattung entrimmt $\mathfrak{B e r}$ fajier namentlich feinem Spejialgebtet. S̃n Den corpora pedunculata bes Gsefirns beliben bie 2 meijen ein fpezielles Seelenorgan, das je nach

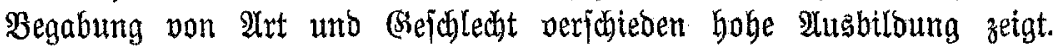
Mit $\mathfrak{A}$ tinnahme Des noch zweifelhaften Bebörorgans baben fie biejelben Sinne mie mir. Shre Reiftung aber unterficheibet fich von ber ber unjeren.

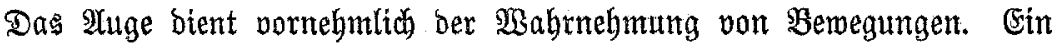
rubender Gegenitand wirb onm ruhenben Snjeft nur unbeutlich ertannt und fein $\mathfrak{B i l d}$ fetwinbet bald ganz. Diejer Mangel wirb jeboch reichlich atseglichen burch ben (Gerudjs= ober beffer Ântennenfinn, ber in $\mathfrak{B}$ er=

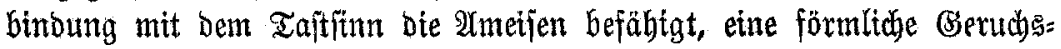

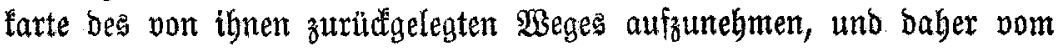

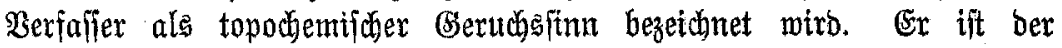
widhtigfte Sinn ber Ilmeifen uno liefert ben Shlüffel zannt Berftänonis

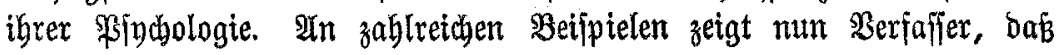




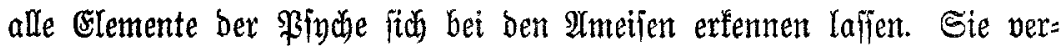

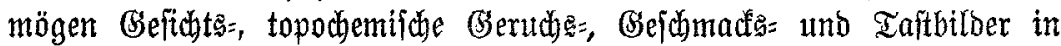
ibrent Sirn zu billoen, zu $\mathfrak{B a h m e h m u n g e n ~ z u ~ f o m b i n i e r e n , ~ u n d ~ b i e ~ b u r c h ~}$

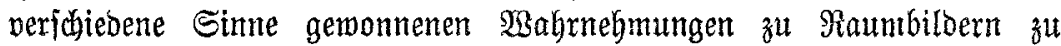

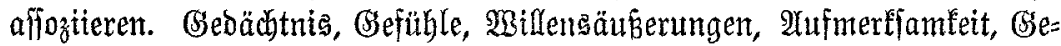
wöhunng uifw. laffen fich mit Beftimmtheit nadheijen, ja ein gewiffes, wenn auch infitinftio ausgeführtes uno veritandenes Miitteilungsvermögen fann nicht in Nbrebe geftellt werben. Wber alle bieje Fäbigkeiten äuß̉ern

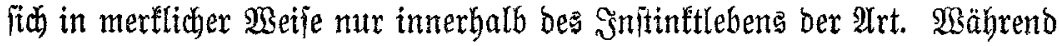

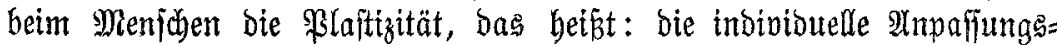
fähiglett an neue $\mathfrak{B e r h a ̈ l t n i f f e ~ e i n e ~ u n g e h e u e r e ~ E n t m i d ł l u n g ~ e r f a h r e n ~ h a t , ~}$

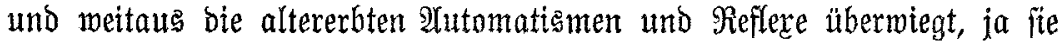
zum Teil unter ihre Serridaft zomingt, bildet bei ben iozialen S̈njeften

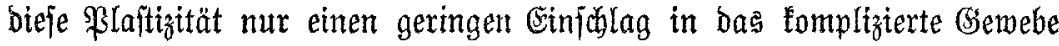
ber erblich firierten geffere uno Intomatismen, die igrem Reben ben Unichein hober S̈ntelligenz verleiben. Forel bält es jogar für jebr zweifelhaft, ob im Şim ber IImeijen überhaupt die einfachften allgemeinen Borftellungen wie Reft, Freund, Feind ufw. auftommen fönnen.

Wie man fith aud zu ben Sdhluffolgerungen ftellen mag, jebenfalle

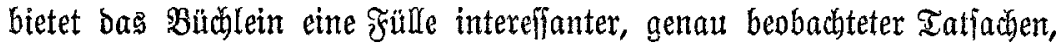
unb regt zu eigenem $\mathfrak{N a b j e n f e n ~ a n . ~ E s ~ f a n n ~ b a b e r ~ a l l e n ~ T i e r f r e u n d e n ~}$ nur auffs wärmite empfoblen werben.

Dr. S.

$\mathfrak{R}, 1 \%$

\section{Menfentajeln zur Beftinumung bes \$olzgehaltes ftehenber}

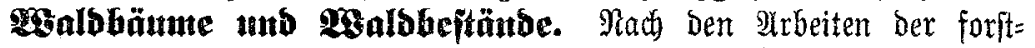

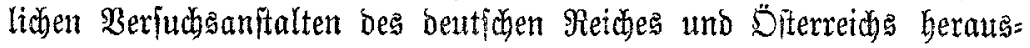
gegeben von Sammerrat Dr. Girunoner unb Prof. Dr. Sdiwappady.

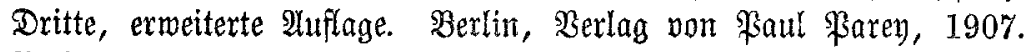

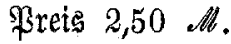

Şm Sabre 1906 exidienen bie Maijentafeln unter obigem Titel in

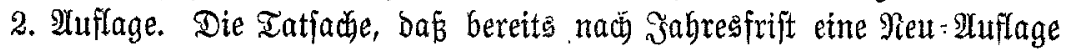
notwendig wurbe, zeigt, wie fehr bie Sherauggeber mit ihren Maffen= tafeln einem Bedürfnis entgegen gefommen fitro. Bu ben bereits in ber

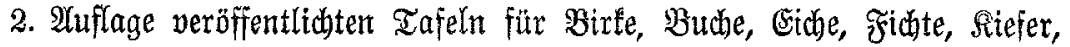

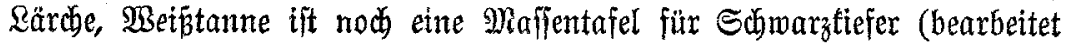

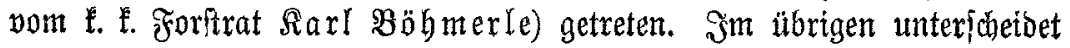
fiti) bie 3. IUflage wenig von ber 2 ten, auf beren Befprechung im Sabr= gang 1906 biejer Beitjarift (ङ. 596) wohl vermiefen werben barf.

Dr. હdüpfer. 
গix. 18.

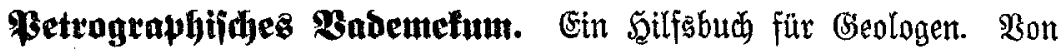

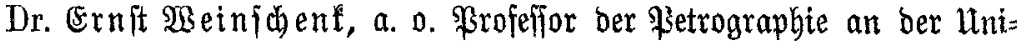
verfität München. Mit einer Tafel und 98 Ibbilbungen. Freiburg,

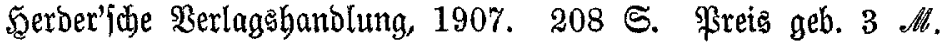

Raut Borwort ift bas von ber Berlagshandlung febr bübjh aus:

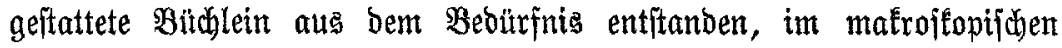
Fraftifum und auf geologifiden Exturfitonen ein Şilfsmittel an ber

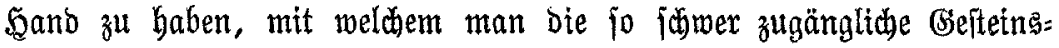
welt für den Bebari des Alugenblicfs etwas überiehen lann. Es foll

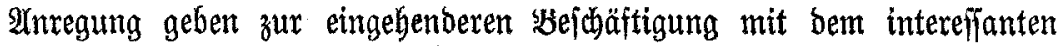
und fo viel vernadläffigten Gebiet der Befteinstunde, nicht etwa ein Sehrbutd) berjelben erieben, wie foldhes ber ßBerfaffer in feinen 2 bänoigen "Srunoziligen ber BSefteingfunde" Darbietet.

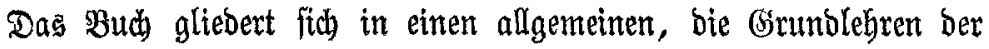
Betrographie bietenden, und einen fpeziellen $\mathfrak{I} e$ il, in welchem bie Gefteine nach ben brei ફ口auptgmppen: Eruptiogeiteine, Sebimentgejteine uno lriftallinifde Schiefer bejprochen werben. Durd zahlreiche atbbildungen miro bas ßeritänonis des im Tert Gebotenen unterftüht.

I.

Rx. 19.

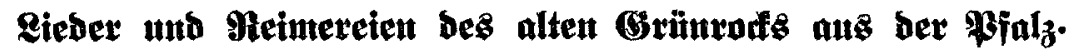

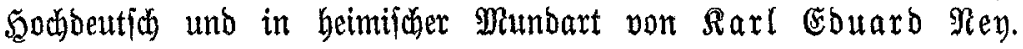

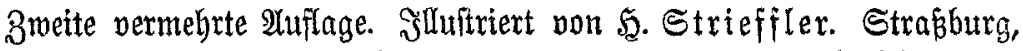
Berlag von Sarl $\mathfrak{3}$. Teubner, 1907. 220 S. Frrets brold. 3 Al.

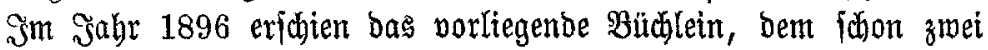

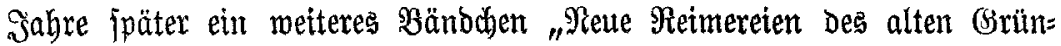
rodfs" folgte, beibe Runoe gebend wie von ber gut beutichen Beftinnung bes in weiteiten Rreifen befannten Berfaffers, fo aud von feinem unverwirtliden Sumor, unb beibe barum aud in forftlichen, wie anderen Ireifen freunblidfit aufgenommen. Der erften Iluflage bes oben genanntert Büchleins ift mun bie zweite gefolgt, vermehrt burd) eime Eleine $\mathfrak{Z n z a h l}$

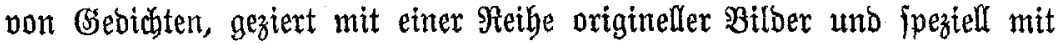
bem Bruftbild bes Serfaffers, bas Seber im 3ujammenthalt mit bem neuen Sebidht "Mein Subdenag" mit ftillem Schmunzeln begrïpen wiro.

Wir wünjön aud ibr weile Berbreitung!

F. 